\title{
Avaliação das propriedades de argamassas cimentícias com adição de resíduos da geração termoelétrica
}

\author{
Evaluation of the properties of cementitious \\ mortars with the addition of thermoelectric \\ generation residues
}

\footnotetext{
${ }^{1}$ Universidade Federal do Pampa (UNIPAMPA), Programa de Pós Graduação em Ciência e Engenharia de Materiais (PPCEM), Grupo de Estudo Avançados em Engenharia de Energia (GREEn), Avenida Maria Anunciação Gomes de Godoy, 1650, CEP 96413-172, RS, Bagé, Brasil.

e-mail:danielelopes.aluno@unipampa.edu.br, sabrinasilva@unipampa.edu.br
}

\section{RESUMO}

A substituição parcial de cimento por resíduos sólidos da geração termoelétrica, em formulações de argamassas, pode contribuir para preservação ambiental e para o desenvolvimento materiais de construção mais duráveis. Este estudo teve como objetivo avaliar a influência da substituição parcial de cimento Portland por cinzas volantes de carvão mineral e pelo subproduto da dessulfuração dos gases de combustão, nas propriedades de argamassas cimentícias. A metodologia experimental consistiu na caracterização dos resíduos por difração de raios-X, espectroscopia Raman, microscopia eletrônica de varredura e análise granulométrica das partículas. Foram preparados blocos cilíndricos de argamassa com dimensão de 50 x $100 \mathrm{~mm}$ e traço 1: 3: 0,48 (cimento: areia: água/cimento), conforme a NBR 7215. O cimento foi substituído, em massa, nas proporções de $6 \%, 16 \%$ e $26 \%$. As resistências à compressão (NBR 7215) foram medidas aos 7, 14 e 28 dias de cura úmida. Complementarmente, realizou-se o ensaio de absorção de água por capilaridade (NBR 9722). Os resultados foram comparados com uma amostra de referência, isto é, sem os resíduos. Foi demonstrado que, mineralogicamente, as cinzas contêm mulita, quartzo (principalmente) e hematita e têm diâmetro médio de $42,19 \mu \mathrm{m}$. A morfologia das partículas é predominantemente esférica contendo algumas porosidades. O subproduto é um material rico em sulfato de cálcio e traços de quartzo com diâmetro médio de $17,8 \mu \mathrm{m}$ sendo constituído por partículas aglomeradas com morfologia variada. Os resultados mostraram que a substituição de $6 \% \mathrm{em}$ massa de cimento Portland por cinzas melhora o desempenho das argamassas quanto à resistência à compressão axial e absorção de água por capilaridade. Para as argamassas contendo o subproduto a melhoria nas propriedades mecânicas não foi evidenciada.

Palavras-chave: Cinza de carvão mineral. Dessulfurização gasosa. Reciclagem. Geração termoelétrica. Argamassas cimentícias.

\begin{abstract}
The partial cement replacement by solid wastes of thermoelectric generation in mortar formulations can contribute to environmental preservation and in a more durable materials building development. This study aimed to evaluate the influence of Portland cement partial replacement by coal fly ash and flue gas desulphurization by-product on the cementitious mortars properties. The experimental methodology consisted of the residues characterization by X-ray diffraction, Raman spectroscopy, scanning electron microscopy and particle size analysis. Cylindrical blocks of mortar with a 50 x $100 \mathrm{~mm}$ dimensions and a 1: 3: 0.48 trace (cement: sand: water/cement) were prepared according with NBR 7215 standard. Cement was replaced, by mass, in the proportions of $6 \%, 16 \%$ and $26 \%$. Comprehensive strengths (NBR 7215) were measured at 7, 14 and 28 days of wet cure. In addition, water absorption by capillarity test was performed (NBR 9722). The results were compared with a reference sample, that is, without residues. It has been shown that, mineralogically, the ashes contain mulita, quartz (mainly) and hematite and have $42.19 \mu \mathrm{m}$ average diameter. Particle
\end{abstract}


morphology is predominantly spherical containing some porosities. The by-product is a material rich in calcium sulfate and quartz traces with $17.8 \mu \mathrm{m}$ average diameter and consists of agglomerated particles with varied morphology. The results showed that the replacement of $6 \%$ Portland cement by ashes improves the performance of mortars in terms of axial comprehensive strength and capillarity water absorption. For the mortars containing the by-product, the improvement in mechanical properties was not evinced.

Keywords: Coal fly ash. Gas desulfurization. Recycling. Thermoelectric generation. Cement mortars.

\section{INTRODUÇÃO}

Concretos e argamassas são os materiais mais utilizados em construções pela sua versatilidade e facilidade de manuseio. A argamassa pode ser conceituada como um material obtido a partir da mistura, em proporções adequadas, de materiais inertes de baixa granulometria e de uma pasta com propriedades aglomerantes. A argamassa diferencia-se do concreto pelo tamanho do agregado que a compõe [1].

As construções podem apresentar problemas estruturais ao longo do tempo. Essa informação é confirmada ao se analisar o número elevado de reconstruções e/ou reparos nas estruturas, decorrentes de falhas prematuras em serviço, agravada pela exposição a atmosferas agressivas. A diminuição na durabilidade de uma estrutura gera um problema ambiental devido ao desperdício de matérias-primas, ao aumento de resíduos gerados, e ao maior consumo de energia e recursos naturais [2, 3].

Nesse contexto, ao longo do tempo, os materiais cimentícios foram se aprimorando pela introdução de aditivos, na forma de plastificantes, retardadores de pega, entre outros, além das adições pozolânicas ou cimentantes que compõem a mistura pela substituição do cimento ou somando-se a este. Assim, estudar soluções que prolonguem a vida útil das estruturas e minimizar o impacto ambiental gerado por sua cadeia produtiva, é um desafio para os pesquisadores e alvo de pesquisas relacionadas ao setor da construção civil [4, 5].

A adição de resíduos nas formulações de argamassas cimentícias já foi evidenciada na literatura. As principais vantagens são os ganhos de resistência, mesmo quando se diminui a quantidade de cimento na mistura. Na microestrutura, contribuem para o desenvolvimento de uma matriz mais definida e uma estrutura menos porosa, o que permite maior resistência ao ataque de agentes agressivos, melhorando sua condição de durabilidade aliada a uma maior resistência mecânica. Dentre as adições, pode-se destacar os resíduos de construção e demolição gerados pela própria construção civil [6], a fibra de coco [7], a cinza de casca de arroz [8], a cinza de bagaço de cana-de-açúcar [9] e as cinzas volantes de carvão mineral [10-12].

Quanto à influência do tempo de cura, a literatura mostra que, aos 28 dias, as argamasssas contendo adições de cinzas de carvão mineral apresentaram melhor desempenho no ensaio de resistência à compressão axial. Isso significa, que não há incremento das reações, no sentido contribuir para de elevar o desempenho das argamassas em tempos de cura superiores aos 28 dias [13-15].

No Brasil, a queima de carvão mineral para a geração de energia em Usinas Termelétricas (UTE’s), gera um montante da ordem de 3,24 milhões de toneladas/ano de resíduos sólidos, tais como escórias, cinzas de carvão mineral (leve e pesada) e o subproduto da dessulfurização dos gases da combustão, processo conhecido como Flue Gas Desulfurization (FGD). Somente uma pequena fração dos subprodutos gerados em UTE's, cerca de $30 \%$, é reaproveitada, o restante é disposto de maneira inadequada. O desenvolvimento de tecnologias de reaproveitamento desses subprodutos, além de minimizar a disposição em áreas inadequadas incrementa a credibilidade deste tipo de geração perante o mercado consumidor [16, 17].

$\mathrm{O}$ alto volume dos resíduos em questão e a potencial atividade pozolânica das cinzas de carvão, comprovada por diversos pesquisadores [16-19], indicam adequabilidade para uso em matrizes cimentícias.

Para ALTHEMAN et al. [17], a adição de cinzas volantes de carvão mineral em matrizes cimentícias é essencial para se alcançar os requisitos da Política Nacional dos Resíduos Sólidos. Os resultados encontrados pelos autores demonstraram que a cinza possui atividade pozolânica e quando inserida nas matrizes cimentícias estudadas, elevaram a resistência mecânica. Os autores desenvolveram argamassas com teores de substituição, em massa, de 5\%, $10 \%$ e $20 \%$ de cimento Portland por cinzas de carvão mineral da produção de alumínio.

PACHECO et al. [18] afirmam que teores de substituição entre 40 e $50 \%$ em massa de cimento Portland por as cinzas volantes de carvão mineral, apresentam reações químicas de hidratação mais lentas, contribuindo pouco para as resistências iniciais, contudo, indicam o material devido à redução do consumo de clínquer.

DUARTE et al. [19] realizaram um estudo que teve por objetivo avaliar o comportamento do concreto, em estado endurecido, com substituições parciais de cimento Portland por cinzas de carvão mineral de 
termoelétricas nos teores de $7 \%, 14 \%$ e $21 \%$, em relação à massa do cimento, e um concreto de referência. Foram realizados ensaios de compressão axial; absorção de água por capilaridade e por imersão na idade de 28 dias. Foi concluído que a resistência à compressão axial diminui conforme aumentam-se os teores de cinzas adicionadas à mistura. Contudo, os valores estão acima da resistência mínima de 20MPa, recomendada pela norma NBR6118. A adição das cinzas não afetou a absorção de água por imersão e por capilaridade.

SANTANA [20], avaliou o uso do subproduto da FGD como adição mineral em concretos com traço de fck (feature compression know) de $25 \mathrm{MPa}$ com 0\%, 5\%, 10\% e 20\% em relação à massa de cimento. Foi demonstrado que as resistências mecânicas dos traços de concreto, ou não sofreram influência ou aumentaram com o aumento do teor de resíduo adicionado.

Com base no disposto pela literatura aqui apresentada, o presente trabalho teve por objetivos caracterizar a cinza volante e o subproduto da FGD, oriundos de uma UTE localizada na cidade de CandiotaRS e, avaliar a influência da substituição de cimento Portland por estes resíduos nas propriedades de argamassas cimentícias.

\section{MATERIAIS E MÉTODOS}

\subsection{Materiais}

Um cimento classificado como CP IV-32 de acordo com a NBR 5736 [21] foi utilizado em todas as formulações. Optou-se por este CP porque é adequado para construção de estruturas enterradas no solo. Além disso, na região da Campanha gaúcha, local deste estudo, estão localizadas UTE’s e, dessa forma, a atmosfera regional possui altas concentrações de sulfatos.

As caracterizações química e física do cimento foram fornecidas pelo fabricante e são mostradas nas Tabelas 1 e 2.

Tabela 1: Composição química do cimento Portland CP IV-32.

\begin{tabular}{c|c|c}
\hline ENSAIOS & RESULTADOS & REFERÊNCIAS \\
\hline Perda de Fogo $-\mathrm{PF}$ & $3,34 \%$ & \multirow{2}{*}{ NBR NM 18 (ABNT, 2004) [22] } \\
\hline Dióxido de Silí́cio $-\mathrm{S}_{\mathrm{i}} \mathrm{O}_{2}$ & $28,94 \%$ & \multirow{3}{*}{ NBR NM 11-2 (ABNT, 2004) [23] } \\
\cline { 1 - 2 } Óxido de Alumínio $-\mathrm{Al}_{2} \mathrm{O}_{3}$ & $9,97 \%$ & \\
\cline { 1 - 2 } Óxido de Ferro $-\mathrm{Fe}_{2} \mathrm{O}_{3}$ & $3,72 \%$ & \\
\cline { 1 - 2 } Óxido de Magnésio $-\mathrm{M}_{\mathrm{g}} \mathrm{O}$ & $3,18 \%$ & \\
\hline Óxido de Cálcio $-\mathrm{C}_{\mathrm{a}} \mathrm{O}$ & $45,12 \%$ & NBR NM 15 (ABNT, 2004) [24] \\
\hline Resíduos Insolúveis - R.I. & $25,25 \%$ & NBR NM 13 (ABNT, 2004) [25] \\
\hline Óxido de Cálcio Livre & $0,74 \%$ &
\end{tabular}

Tabela 2: Caracterização física do cimento Portland CP IV-32.

\begin{tabular}{c|c|c}
\hline ENSAIOS & RESULTADOS & REFERÊNCIAS \\
\hline Massa específica & $2,83 \mathrm{~g} / \mathrm{cm}^{3}$ & NBR NM 23 (ABNT, 2001) [26] \\
\hline Área específica & $4340 \mathrm{~cm}^{2} / \mathrm{g}$ & NBR NM 76(ABNT, 1998) [27] \\
\hline Finura Blaine & $4,290 \mathrm{~cm}^{2} / \mathrm{g}$ & NBR NM 11579(ABNT, 1991) [28] \\
\hline Água da pasta de consistência normal & $30,3 \%$ & NBR NM 43(ABNT, 2003) [29] \\
\hline Início de pega & $3,4 \mathrm{~h}$ & \multirow{2}{*}{ NBR NM 65(ABNT, 2003) [30] } \\
\hline Fim de pega & $4,25 \mathrm{~h}$ & \multirow{2}{*}{ NBR NM 11582(ABNT, 1991) [31] } \\
\cline { 1 - 2 } Expansividade de Le Chatelier - a quente & $0,34 \mathrm{~mm}$ & \multirow{2}{*}{ NBR 7215 (ABNT, 2019) [32] } \\
\cline { 1 - 2 } Resistência à compressão 1 dia & - & \\
\cline { 1 - 2 } Resistência à compressão 3 dias & $>10,0 \mathrm{MPa}$ & \\
\cline { 1 - 2 } Resistência à compressão 7 dias & $>20,0 \mathrm{MPa}$ & \\
\cline { 1 - 2 } Resistência à compressão 28 dias & $>32,0 \mathrm{MPa}$ &
\end{tabular}

O agregado miúdo utilizado foi a areia fina com tamanho máximo de partícula de $1 \mathrm{~mm}$ (NBR 7211 [33]). A água de amassamento foi proveniente da rede de esgoto local da cidade de Bagé-RS.

O CP foi parcialmente substituído, em massa, por cinzas volantes (CV) e pelo subproduto da FGD 
(SP) cedidos pelo Complexo Termelétrico de Candiota. Os resíduos não tiveram nenhum tipo de preparo, manipulação preliminar à adição na argamassa.

\subsection{Caracterização dos materiais}

A composição mineral dos resíduos foi determinada por difratometria de raios-X (DRX) e espectroscopia Raman, tais técnicas possuem alta resolução para identificação de minerais mesmo em baixas concentrações. Ambas as técnicas fornecem informações sobre os compostos, contudo, pela primeira identificam-se também as estruturas cristalinas. A combinação das técnicas é útil nos casos em que a DRX não fornecer informações estruturais satisfatórias, devido, por exemplo, a elevada concentração de material vítreo [34].

O difratrômetro utilizado foi o Rigaku ULTIMA IV, JP. Operando com radiação de $\mathrm{Cu} \mathrm{K} \alpha$, gerada a 40 $\mathrm{kV}$ e $20 \mathrm{~mA}$. A amplitude de escaneamento foi de $3^{\circ}$ a $80^{\circ}$ e tempo de integração de 2 s por ponto.

Os espesctros Raman foram medidos em um espectrômetro Raman Confocal com laser de $(\lambda=532 \mathrm{~nm})$, e diâmetro de feixe de $1 \mu \mathrm{m}$ no intervalo de 5 a $1900 \mathrm{~cm}-1$.

Os espectros experimentais foram comparados com a base de dados RRUFF (http://rruff.info/) [35] e as fases minerais foram identificadas com auxílio do software X-pert High Score Plus.

A morfologia das partículas, analisadas por microscopia eletrônica de varredura (MEV) foi analisada em um microscópio da marca Jeol, JSM - 6610LV.

Pela análise granulométrica, NBR 7181 [36], avaliou-se adispersão de tamanhos e o diâmetro médio das partículas. O ensaio foi realizado em um em granulômetro a laser modelo 1190 LD, da marca CILAS, medido em solução líquida.

\subsection{Formulações e preparo dos corpos de prova}

Os corpos de prova foram preparados com argamassa composta por uma parte de cimento, três de areia normalizada, em massa, e com relação água cimento de 0,48 (1:3:0,48), conforme a NBR 7215 [32].

Foram preparados corpos de prova cum teores de substituição, em massa, de CP por CV ou SP de 0, $6 \%, 16 \%$ e $26 \%$. A Tabela 3 apresenta as formulações dos traços analisadas na pesquisa.

Tabela 3: Traço de referência e com CV e SP.

\begin{tabular}{|c|c|c|c|c|c|c|c|}
\hline \multirow{2}{*}{ TRAÇO } & \multicolumn{3}{|c|}{ MASSA DE AGLOMERANTE } & \multirow{2}{*}{ AREIA (g) } & \multirow{2}{*}{$\begin{array}{l}\text { ÁGUA } \\
(\mathrm{mL})\end{array}$} & \multirow{2}{*}{$\begin{array}{c}\text { RELAÇÃO } \\
\text { água/cimento }\end{array}$} & \multirow{2}{*}{$\begin{array}{c}\text { Consumo de cimento } \\
\left(\mathrm{kg} / \mathrm{m}^{3}\right)\end{array}$} \\
\hline & CIMENTO (g) & CV $(g)$ & SP (g) & & & & \\
\hline $0 \%$ & 624 & - & - & 1872 & 300 & 0,48 & 3120 \\
\hline $6 \%$ & 586,56 & 37,44 & - & 1872 & 300 & 0,48 & 2932,8 \\
\hline $16 \%$ & 524,16 & 99,84 & - & 1872 & 300 & 0,48 & 2620,8 \\
\hline $26 \%$ & 461,76 & 162,24 & - & 1872 & 300 & 0,48 & 2308,8 \\
\hline $6 \%$ & 586,56 & - & 37,44 & 1872 & 300 & 0,48 & 2932,8 \\
\hline $16 \%$ & 524,16 & - & 99,84 & 1872 & 300 & 0,48 & 2620,8 \\
\hline $26 \%$ & 461,76 & - & 162,24 & 1872 & 300 & 0,48 & 2308,8 \\
\hline
\end{tabular}

A Figura 1 mostra as argamassas nos moldes antes da cura úmida (1a) e as mesmas desmoldadas após 24 horas de cura (1b).

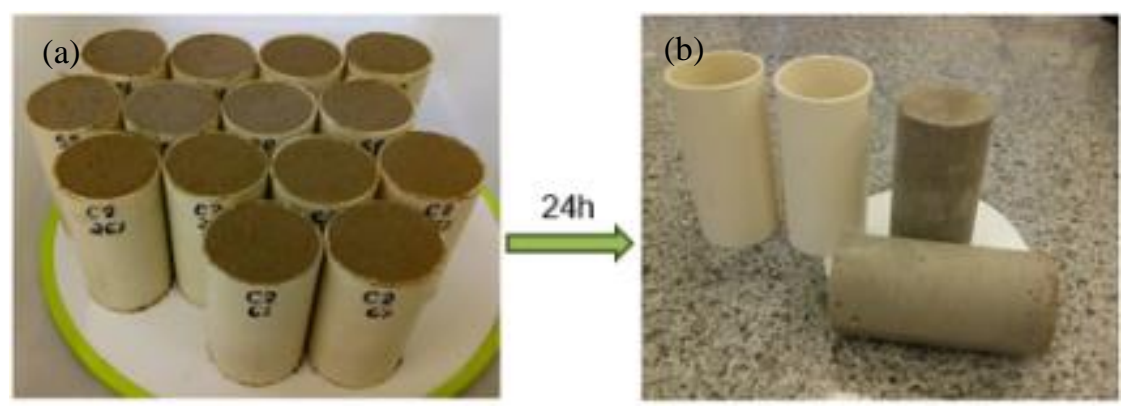

Figura 1: Corpos de prova nos (a) moldes e (b) desmoldados após 24 horas. 


\subsection{Resistência à compressão}

O método de ensaio foi realizado conforme o prescrito pela NBR7215 [32] o qual compreende a determinação da resistência à compressão em corpos de prova cilíndricos de $50 \mathrm{~mm}$ de diâmetro e $100 \mathrm{~mm}$ de altura. Utilizou-se um equipamento da marca EMIC modelo DL 20000 com velocidade de aplicação da carga de $0,45 \pm 0,15 \mathrm{MPa} / \mathrm{s}$ e carga máxima de $100 \mathrm{kN}$. As amostras foram comprimidas até a ruptura nas idades de 7 , 14 e 28 dias de cura úmida.

\subsection{Determinação da absorção de água por capilaridade}

Este ensaio é útil para monitorar o aumento da massa das argamassas devido à absorção de água por capilaridade conforme a NBR 9779 [37]. A absorção de água por capilaridade foi calculada pela equação 1:

$$
C=(A-B) / S
$$

Onde: $C$ é a absorção de água por capilaridade em $\mathrm{g} / \mathrm{cm}^{2}$; A é a massa do corpo de prova que permanece com uma de suas faces em contato com a água; B é a massa do corpo de prova seco e $\mathrm{S}$ é a área da seção transversal em $\mathrm{cm}^{2}$.

\subsection{Análise estatística}

Para verificar se existem influências significativas das substituições na variável resposta resistência à compressão, utilizou-se a análise de variância (ANOVA), com delineamento inteiramente casualizado (DIC).

\section{RESULTADOS E DISCUSSÃO}

No difratograma da CV, apresentado na Figura 3a, destacou-se um halo amorfo entre $15^{\circ}$ e $35^{\circ}$ passível de atividade pozolânica deste resíduo, conforme indicam HOPPE et al. [38] e YAN et al. [39].

$\mathrm{Na}$ composição mineralógica da $\mathrm{CV}$, foram identificadas fases de quartzo $\left(\mathrm{S}_{\mathrm{i}} \mathrm{O}_{2}\right)$, mulita $\left(\mathrm{Al}_{2} \mathrm{O}_{3}-\mathrm{S}_{\mathrm{i}} \mathrm{O}_{2}\right)$ e traços de hematita $\left(\mathrm{Fe}_{2} \mathrm{O}_{3}\right)$ conforme mostram os difratogramas, Figura 3a, e o espectro Raman, Figura $3 \mathrm{~b}$. De acordo com a intensidade dos picos, verifica-se que o quartzo é mais frequente, seguido de mulita e hematita. Esta mineralogia também foi evidenciada por KRÓL et al. [40] e por AZEVEDO et al. [41]. Mesmo sendo de locais distintos, observou-se semelhanças entre os difratogramas principalmente no halo amorfo e no pico de quartzo que fica entre $26^{\circ}$ e $27^{\circ}$ [38-41]. A formação da mulita, identificada somente por DRX, está relacionada com a combustão do carvão em temperaturas maiores do que $1050^{\circ} \mathrm{C}$. A mulita é a fase que contribui para resistência mecânica do material [42].

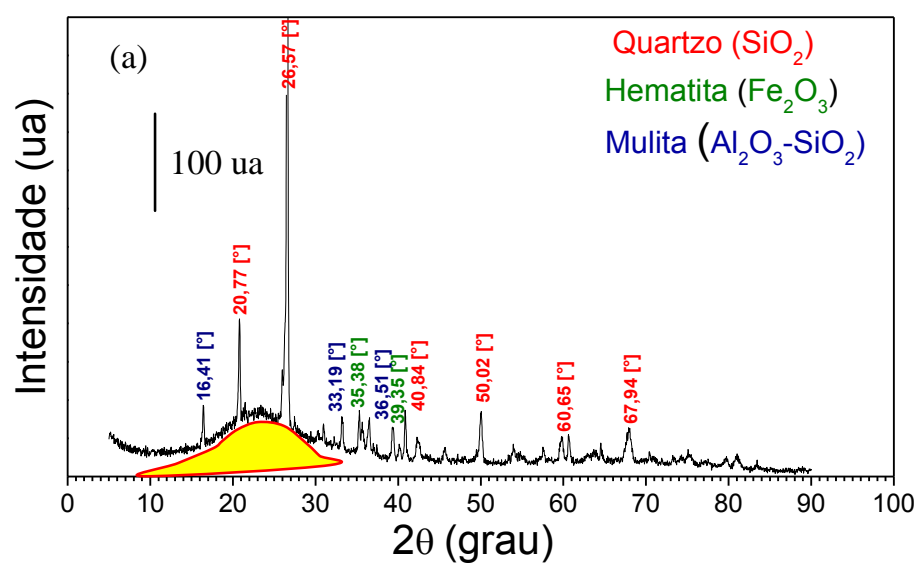




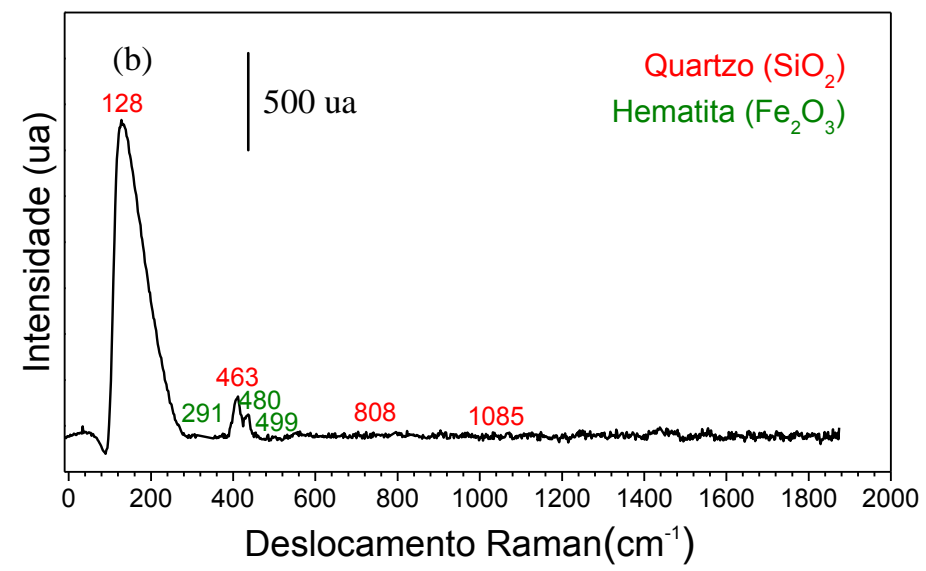

Figura 2: Difratograma (a) e espectro Raman (b) da CV.

Ao analisar o difratograma do SP, observa-se um baixo grau de amorfismo, devido à ausência de halo amorfo e, portanto, o material não tem atividade pozolânica. O material possui uma estrutura bem ordenada, com picos de intensidade bem definidos. Quanto a composição mineral, a fase mais significativa é o sulfato de cálcio $\left(\mathrm{CaSO}_{4} \cdot 2 \mathrm{H}_{2} \mathrm{O}\right)$, na forma de gipsita, e quartzo, conforme mostram as Figuras 3a-b. Portanto, o resíduo não pode ser considerado puro. O quartzo identificado é devido à $\mathrm{CV}$ remanescente que se mistura ao SP durante a dessulfurização. LI et al. [43] e FENG et al. [44] identificaram estes compostos em subprodutos de FGD.
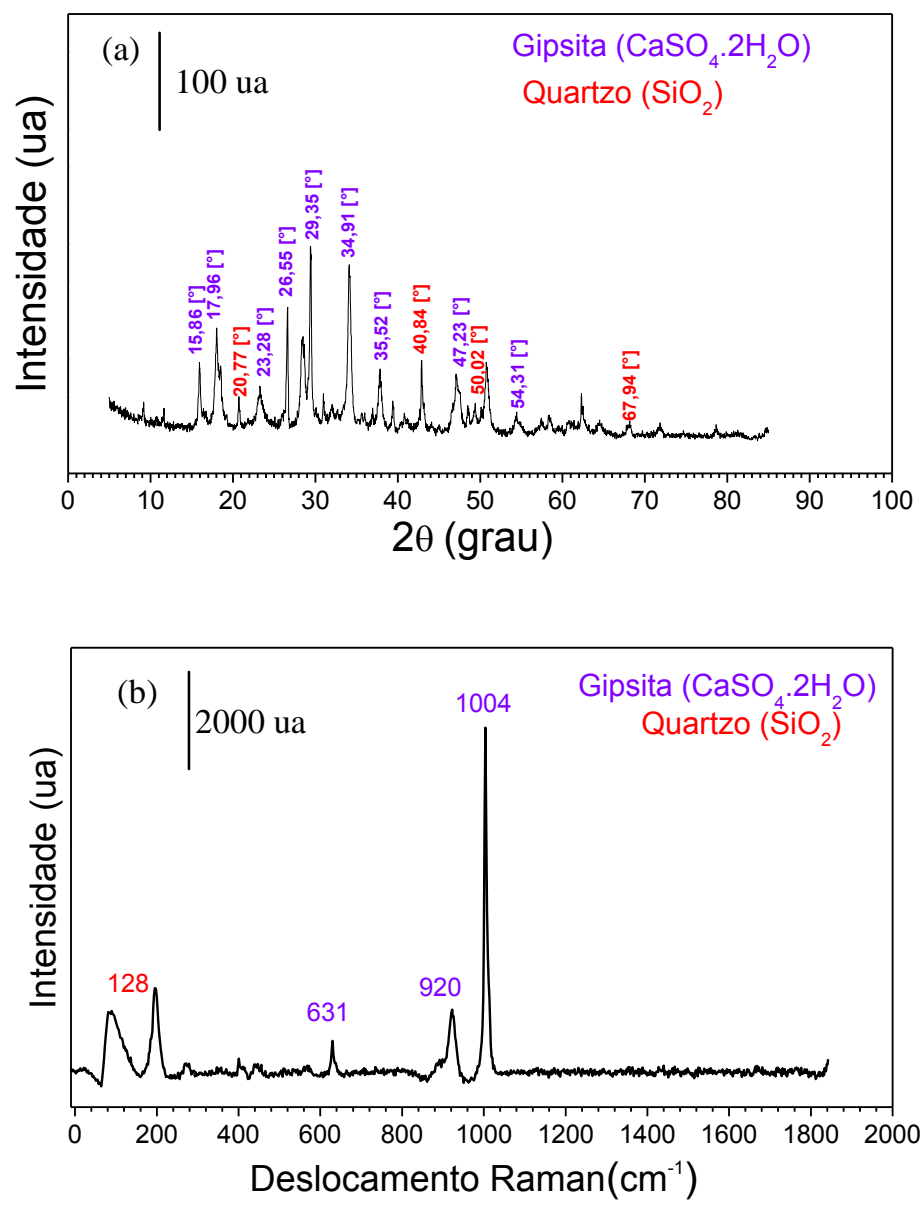

Figura 3: Difratograma (a) e espectro Raman (b) do SP. 
Na micrografia de MEV da CV, Figura 4a, pode-se observar partículas com diâmetro variando entre 0,7 $\mu \mathrm{m}$ e $20 \mu \mathrm{m}$, morfologia esférica com superfície lisa (maioria) e algumas com microporosidades. A morfologia esférica é tipicamente identificada em cinzas volantes provenientes da combustão de carvão pulverizado conforme relatado na literatura [45, 46].

Nas partículas de SP, Figura 4b, observam-se aglomerados com morfologia variada, contendo alguns cristais escamosos e partículas prismático-rômbicas de dimensão variada e distribuição heterogênea, condizentes com a morfologia observada por outros autores [47, 48].

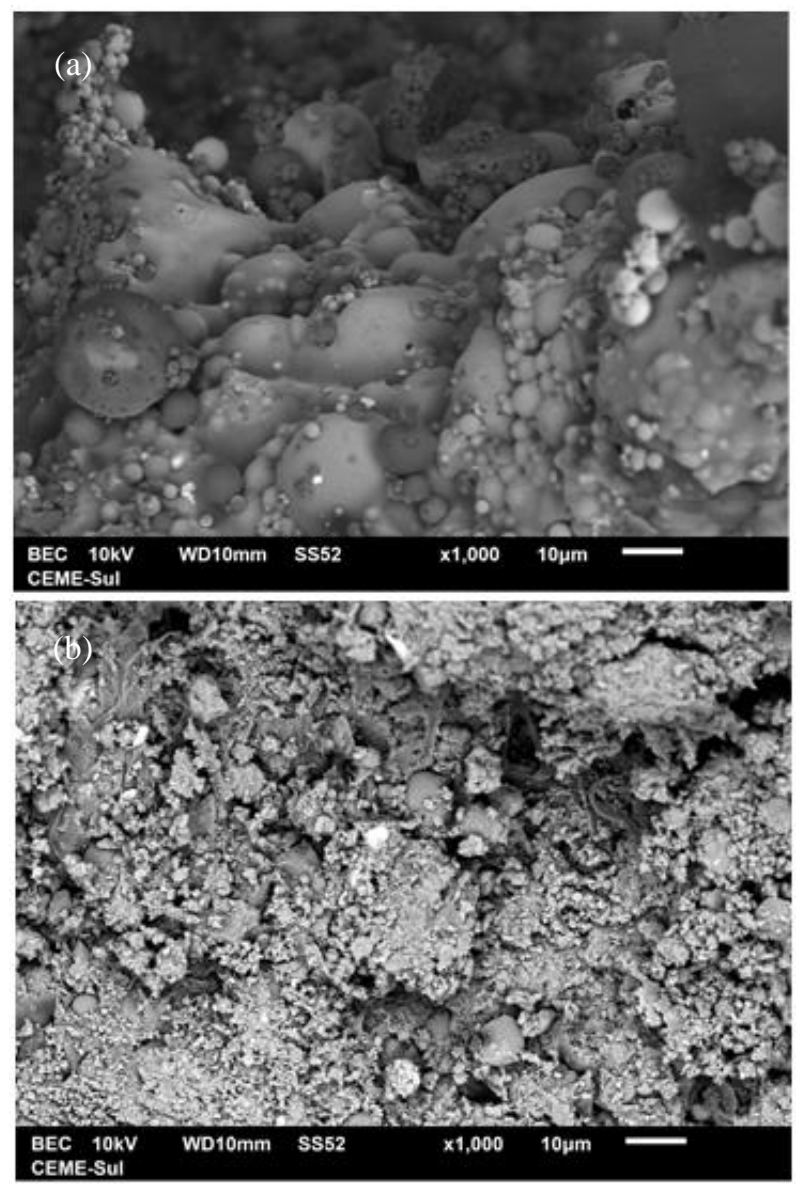

Figura 4: Imagens de MEV (a) CV e (b) SP.

A Figura 5 mostra a distribuição granulométrica dos resíduos. $\mathrm{O}$ eixo $\mathrm{x}$ dos diagramas mostra os diâmetros em $\mu \mathrm{m}$, no eixo y à esquerda, a massa acumulada \% e no eixo y à direita o histograma.

Foram medidos diâmetros médios de $42,19 \mu \mathrm{m}$ e $17,83 \mu \mathrm{m}$ para a CV e para o SP, respectivamente.
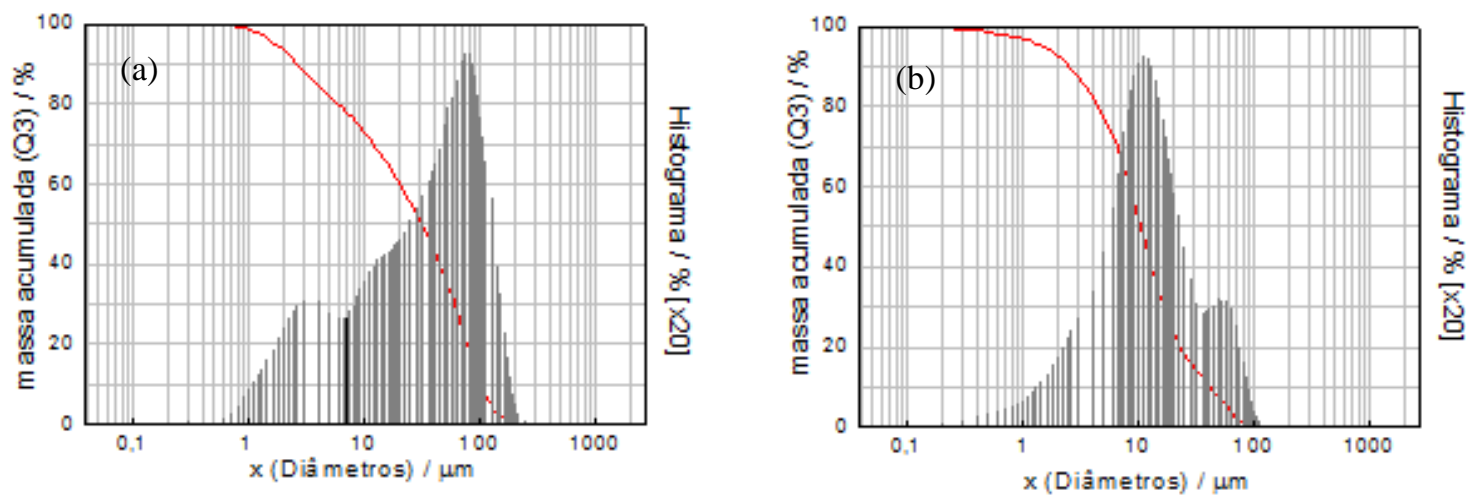
Figura 5: Distribuição granulométrica (a) CV e (b) SP.

Conforme MEHTA e MONTEIRO [49], a cinza volante pode ter diâmetro variando entre $1 \mu \mathrm{m}$ até $100 \mu \mathrm{m}$. COSTA [50] obteve diâmetro médio de 41,35 $\mu \mathrm{m}$, enquanto LACERDA [51], 54,22 $\mu \mathrm{m}$, ambos para cinzas volantes provenientes da combustão do carvão na mesma UTE que forneceu o material para este estudo. As diferenças entre o diâmetro médio encontrado neste trabalho com os de outros autores [50, 51] está relacionada às características do processo de combustão e do carvão mineral queimado [52].

ZERBINO et al. [53] destacam a importância da distribuição granulométrica dos resíduos no que tange ao desempenho da pasta que a incorpora. No caso do concreto contendo cinza, em teor de substituição de $15 \%$ em massa, os autores observaram melhorias nas propriedades do estado fresco e também, nas propriedades mecânicas e na durabilidade. Isso devido à maior finura do material. Ainda conforme ZERBINO et al. [53], a cinza pode, inclusive, influenciar na inibição ou desenvolvimento da reação álcalisílica (RAS). A mitigação ou exacerbação da RAS depende do teor de substituição do cimento e, preponderantemente, do tamanho das partículas do material adicionado.

A Figura 6 mostra os corpos de prova antes do ensaio de resistência à compressão axial (6a), posicionados no equipamento (6b) e rompidos (6c). A seguir, na Tabela 5, são mostrados os valores das resistências à compressão axial dos corpos de prova das argamassas moldadas com diferentes teores de substituição do cimento pelos resíduos após 7, 14 e 28 dias de cura. Estes valores correspondem à média de três medidas. Graficamente, os resultados são mostrados na Figura 7.

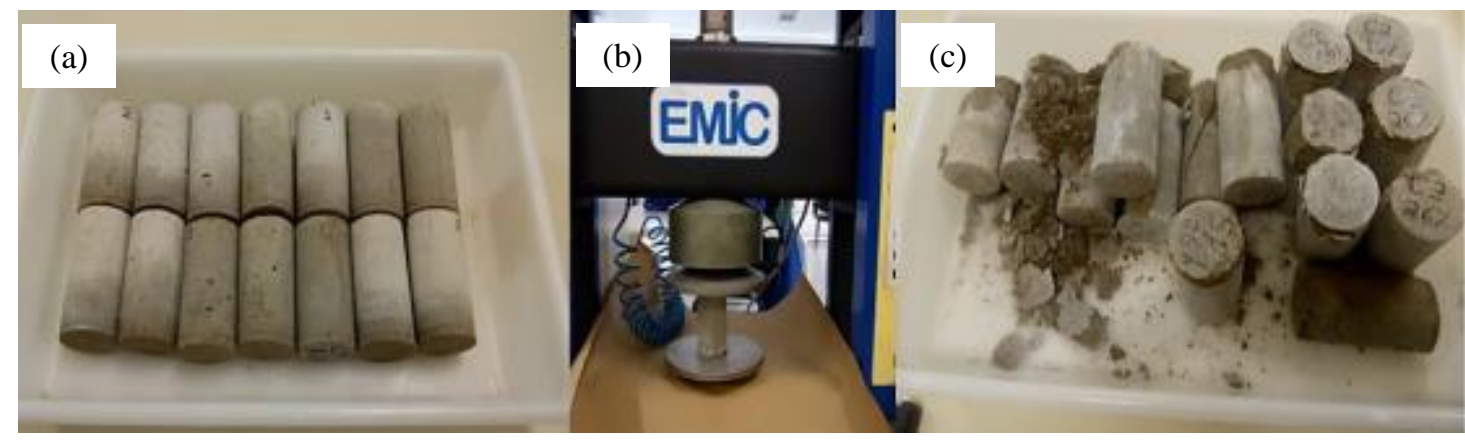

Figura 6: Argamassas (a) antes (b) durante e (c) após o a medida da resistência à compressão.

Tabela 5: Resistência à compressão axial das argamassas cimentícias.

\begin{tabular}{c|c|c|c}
\hline \multirow{2}{*}{ ARGAMASSA } & \multicolumn{3}{|c}{ RESISTÊNCIA À COMPRESSÃO AXIAL (MPa) } \\
\cline { 2 - 4 } & $\mathbf{7}$ dias & $\mathbf{1 4}$ dias & $\mathbf{2 8}$ dias \\
\hline REF & 17,37 & 19,81 & 27,76 \\
\hline $6 \% \mathrm{CV}$ & 18,98 & 24,36 & 29,29 \\
\hline $16 \% \mathrm{CV}$ & 15,27 & 18,99 & 23,97 \\
\hline $26 \% \mathrm{CV}$ & 12,98 & 14,11 & 17,58 \\
\hline $6 \% \mathrm{SP}$ & 7,06 & 8,87 & 22,32 \\
\hline $16 \% \mathrm{SP}$ & 6,75 & 8,78 & 21,72 \\
\hline $26 \% \mathrm{SP}$ & 6,89 & 7,22 & 14,88 \\
\hline
\end{tabular}




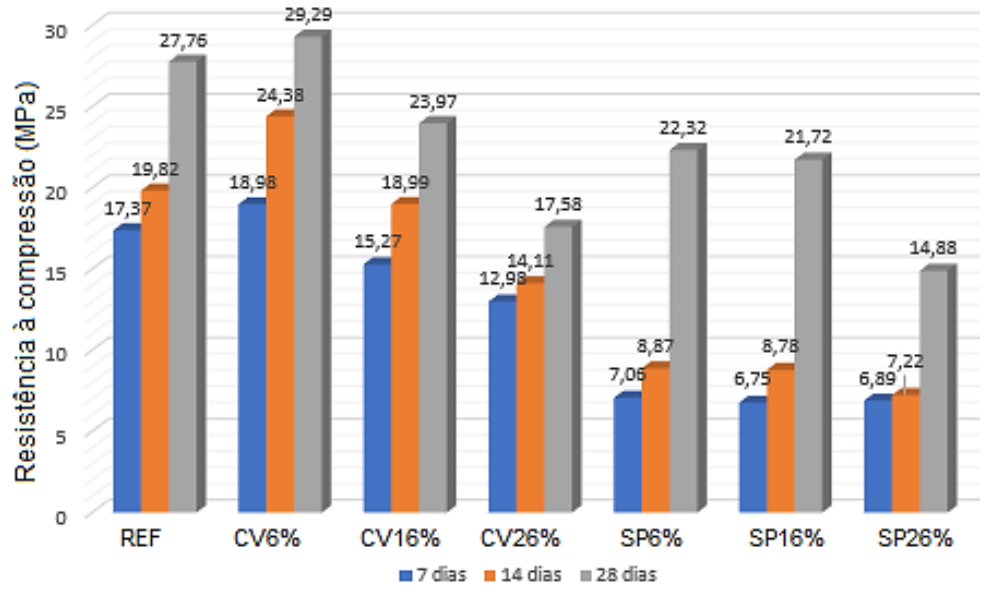

Figura 7: Resistência à compressão axial das argamassas cimentícias.

A análise dos resultados permitiu inferir que, aos 28 dias de cura, as resistências foram mais elevadas. Com relação ao teor de substituição, o ganho maior foi para a argamassa com teor de substituição de cimento por $6 \%$ de CV. Segundo HOANG et al. [54], as resistências iniciais nas idades menores são inferiores devido ao menor conteúdo de clínquer e às reações tardias do material pozolânico. A substituição de cimento por material pozolânico além de seu efeito químico, atua também diminuindo o tamanho dos poros da matriz cimentícia [55]. O ganho de resistência para essa formulação, isto é, com 6\% de CV, foi de 1,53 MPa, esse valor pode ser considerado pequeno, contudo, indica-se o material devido à redução do consumo de cimento de $187,2 \mathrm{~kg} / \mathrm{m}^{3}$.

Em relação dos corpos de prova contendo SP, não houve ganho de resistência nos teores de substituição e tempos de cura analisados neste estudo. No trabalho desenvolvido por KHATIB et al. [56], o qual avaliou a substituição de cimento por resíduo de FGD em concreto nas porcentagens em massa variando entre $10 \%$ a $90 \%$ em tempos de cura de 1, 7, 28 e 365 dias, a adição do resíduo contribuiu para o ganho de resistência à compressão somente para o concreto contendo $10 \%$ de resíduo aos 365 dias de cura. Nas demais condições os resultados foram inferiores ao concreto de referência. Nos ensaios de resistência a flexão, o ganho foi limitado ao teor de substituição de $30 \%$ aos 28 dias de cura. Possivelmente, devido à adição deste resíduo, formam-se produtos expansivos (deletérios), os quais geram tensões internas no material.

Para análise estatística, foram considerados três fatores: tipo de argamassa (A), tempo de cura (B) e o teor de substituição percentual de cimento (C). Assim, o fator A possui dois níveis, o fator B três níveis enquanto o fator $\mathrm{C}$ possui quatro níveis. $\mathrm{O}$ planejamento fatorial foi realizado com duas réplicas. A distribuição dos dados para a análise está apresentada na Tabela 6.

Tabela 6: Dados para análise fatorial geral.

\begin{tabular}{|c|c|c|c|c|c|c|c|c|c|c|c|c|}
\hline \multirow{4}{*}{$\begin{array}{c}\text { TIPOS DE } \\
\text { ARGAMASSA } \\
\text { (A) }\end{array}$} & \multicolumn{12}{|c|}{ Tempo de cura (B) } \\
\hline & \multicolumn{4}{|c|}{7 dias } & \multicolumn{4}{|c|}{14 dias } & \multicolumn{4}{|c|}{28 dias } \\
\hline & \multicolumn{12}{|c|}{ Teor de substituição pencentual de cimento (C) } \\
\hline & $0 \%$ & $6 \%$ & $16 \%$ & $26 \%$ & $0 \%$ & $6 \%$ & $16 \%$ & $26 \%$ & $0 \%$ & $6 \%$ & $16 \%$ & $26 \%$ \\
\hline \multirow{2}{*}{$\begin{array}{c}\text { Contendo } \\
\mathrm{CV}\end{array}$} & \multirow{4}{*}{$\begin{array}{l}16,89 \\
17,85\end{array}$} & 18,25 & 14,99 & 14,83 & \multirow{4}{*}{$\begin{array}{l}19,22 \\
20,42\end{array}$} & 24,60 & 20,42 & 13,69 & \multirow{4}{*}{$\begin{array}{l}27,45 \\
28,07\end{array}$} & 30,26 & 23,20 & 17,21 \\
\hline & & 19,71 & 15,55 & 11,13 & & 24,12 & 17,56 & 14,53 & & 28,32 & 24,74 & 17,96 \\
\hline Contendo & & 7,59 & 5,98 & 6,01 & & 7,99 & 8,02 & 8,12 & & 21,54 & 22,94 & 15,80 \\
\hline SP & & 6,53 & 7,52 & 7,77 & & 9,74 & 9,54 & 6,32 & & 23,10 & 20,50 & 13,96 \\
\hline
\end{tabular}

Com o planejamento fatorial realizado, verificou-se que ocorreu significância nos fatores de primeira ordem (A, B e C) e nos fatores de segunda ordem (AC e BC). Observou-se que o tempo, fator B, tem interação com o traço de argamassa utilizado e com o teor de substituição utilizado (fator C).

Percebeu-se que o tempo influenciava nos resultados, optou-se em realizar esta análise separadamente, usando apenas os fatores B e C. Na Tabela 7 está apresentado os resultados de ANOVA para os a) 7, b) 14 e c) 28 dias de cura úmida. 
Tabela 7: Anova para a) 7 dias b) 14 dias e c) 28 dias.

\begin{tabular}{|c|c|c|c|c|c|}
\hline a) ANOVA (7 Dias) & $\begin{array}{c}\text { Soma } \\
\text { Quadrática }\end{array}$ & $\begin{array}{c}\text { Média } \\
\text { Quadrática }\end{array}$ & $\mathbf{F}$ & P-value & F crit \\
\hline Tipo de argamassa & 175,9602 & 175,9602 & 114,5798 & $5,096 \mathrm{E}-06$ & 5,317655 \\
\hline$\%$ de teor & 129,3643 & 43,12143 & 28,07933 & 0,0001343 & 4,066181 \\
\hline Interação & 75,80467 & 25,26823 & 16,45388 & 0,0008775 & 4,066181 \\
\hline Total & 381,1292 & & & & \\
\hline b) ANOVA (14 Dias) & $\begin{array}{c}\text { Soma } \\
\text { Quadrática }\end{array}$ & $\begin{array}{c}\text { Média } \\
\text { Quadrática }\end{array}$ & $\mathbf{F}$ & P-value & F crit \\
\hline Tipo de argamassa & 265,6085 & 265,6085 & 206,2127 & $5,400 \mathrm{E}-07$ & 5,317655 \\
\hline$\%$ de teor & 182,5067 & 60,8355 & 47,2314 & $1,964 \mathrm{E}-05$ & 4,066180 \\
\hline Interação & 126,2027 & 42,0675 & 32,6603 & $7,744 \mathrm{E}-05$ & 4,066180 \\
\hline Total & 574,3179 & & & & \\
\hline c) ANOVA (28 Dias) & $\begin{array}{c}\text { Soma } \\
\text { Quadrática }\end{array}$ & $\begin{array}{c}\text { Média } \\
\text { Quadrática }\end{array}$ & $\mathbf{F}$ & P-value & F crit \\
\hline Tipo de argamassa & 35,55141 & 35,55141 & 27,40085 & 0,0007885 & 5,317655 \\
\hline$\%$ de teor & 304,982 & 101,6607 & 78,35383 & $2,85 \mathrm{E}-06$ & 4,066181 \\
\hline Interação & 25,40902 & 8,469673 & 6,527906 & 0,0152519 & 4,066181 \\
\hline Total & 365,9424 & & & & \\
\hline
\end{tabular}

No diagrama que relaciona a variação da resistência à compressão com as adições percentuais (Figura 8), verifica-se que a adição de $6 \%$ de CV está associada à resistência média mais alta, indicando que este efeito principal é significativo na variável resposta (ganho de resistência aos 28 dias). Percebe-se também que nas substituições em teores maiores, 16 e 26\%, houve decréscimo da resistência. Os diagramas mostrado na Figura 8 enfatiza que para todas as adições estudadas a CV conferiu maior resistência às argamassas comparando-se com o SP e também que na argamassa contendo $6 \% \mathrm{CV}$ obteve-se a resistência mais elevada do que todas as situações avaliadas.
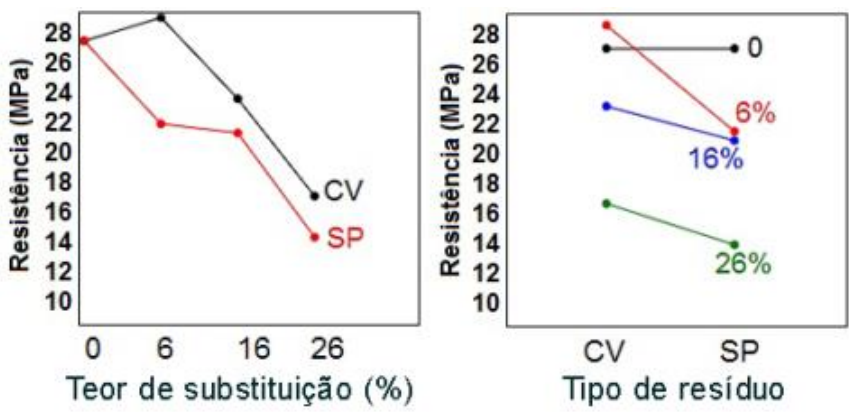

Figura 8: Efeitos das adições na resistência mecânica.

A absorção de água por capilaridade diminuiu para a argamassa com teor de substituição de $6 \%$ de CV. Isto pode ser explicado pelo refinamento dos poros atribuído à adição do resíduo. Contudo, esse valor ainda não atende as prescrições da NBR 16072 [58] que define uma porcentagem mínima de redução de 50\% para que uma argamassa seja considerada impermeável.

Quanto maior o percentual de resíduo, maior a absorção por capilaridade, ou seja, o material torna-se mais permeável e esse comportamento pode ser prejudicial dependendo da aplicação. Além disso, como a relação $(\mathrm{a} / \mathrm{c})$ é baixa, os resíduos tendem a absorver mais água segregando as partículas no interior do material. Essa segregação pode ter sido um dos fatores para o aumento da absorção por capilaridade [59].

A absorção de água por capilaridade pode ainda estar relacionado à tortuosidade dos poros na matriz cimentícia. Neste caso, uma maior tortuosidade dificulta a permeação da água $[59,60]$. 
Segundo BOTAS [61], a capilaridade pode ser analisada em termos de velocidade inicial de absorção e em termos de quantidade total de água absorvida. Argamassas com poros de menores dimensões dão origem a menores velocidades iniciais de absorção, mas a maior quantidade de água absorvida. Por outro lado, a conectividade da rede porosa, assim como, a própria porosidade aberta, são propriedades que condicionam a absorção de água por capilaridade, o que é um dos principais responsáveis pela degradação de revestimentos argamassados.

Os resultados estão apresentados na Tabela 8 e, graficamente, na Figura 9. A ascenção capilar da água nas argamassas é mostrada na Figura 10.

Tabela 8: Absorção de água por capilaridade, em g/ $\mathrm{cm}^{2}$, em função do teor de substituição de cimento por CV ou SP.

\begin{tabular}{c|c|c|c|c|c|c|c}
\hline \multirow{2}{*}{$\begin{array}{c}\text { TEMPO } \\
\text { (horas) }\end{array}$} & \multicolumn{7}{|c}{ ARGAMASSA } \\
\cline { 2 - 8 } & REF & CZ 6\% & CZ 16\% & CZ 26\% & SP 6\% & SP 16\% & SP 26\% \\
\hline $\mathbf{0}$ & 0 & 0 & 0 & 0 & 0 & 0 & 0 \\
\hline $\mathbf{6}$ & 0,37 & 0,32 & 0,501 & 0,41 & 0,43 & 0,47 & 0,56 \\
\hline $\mathbf{2 4}$ & 0,52 & 0,43 & 0,697 & 0,62 & 0,56 & 0,59 & 0,71 \\
\hline $\mathbf{4 8}$ & 0,62 & 0,51 & 0,841 & 0,72 & 0,68 & 0,76 & 0,85 \\
\hline $\mathbf{7 2}$ & 0,63 & 0,53 & 0,913 & 0,76 & 0,72 & 0,79 & 0,93 \\
\hline
\end{tabular}

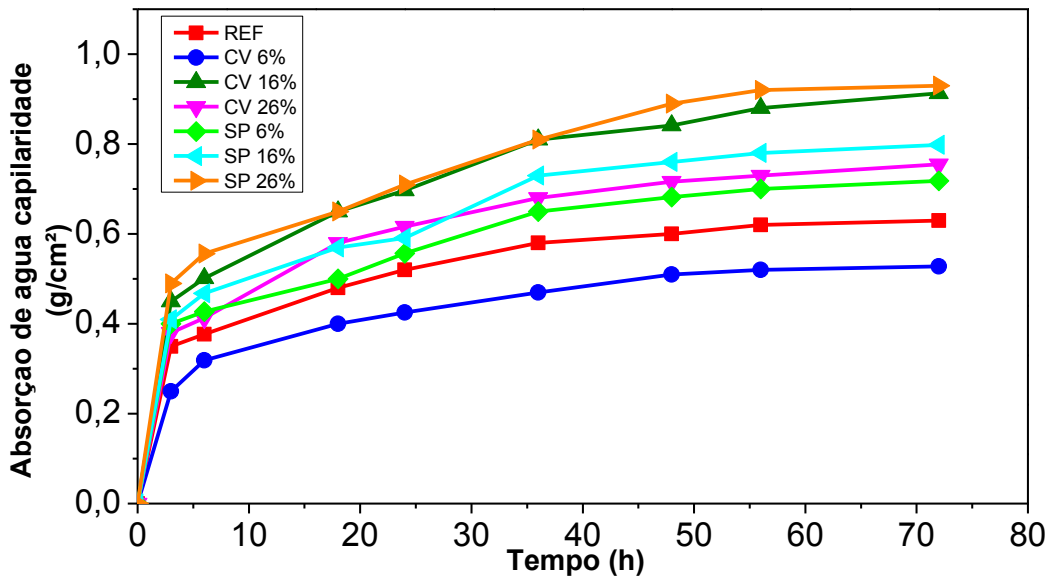

Figura 9: Absorção de água por capilaridade, em g/ $\mathrm{cm}^{2}$, em função do teor de substituição de cimento por CV ou SP.
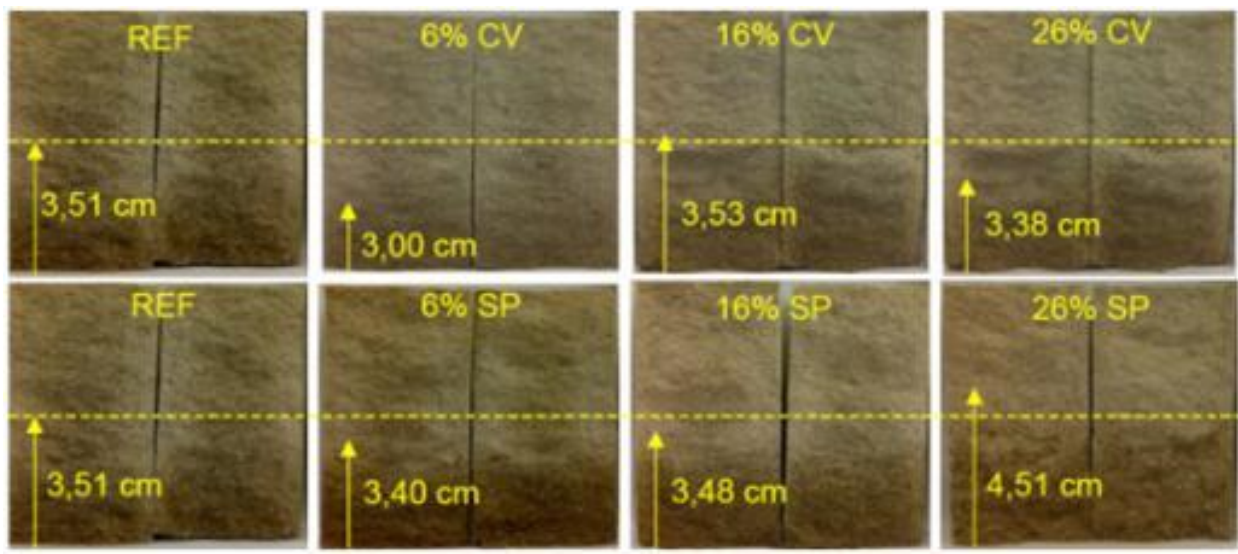

Figura 10: Argamassas cimentícias rompidas diametralmente para aferição da ascensão de água. 


\section{CONCLUSÕES}

Nesse trabalho, argamassas a base de cimento Portland formulados com substituição parcial deste por resíduos da geração termoelétrica. De acordo com os objetivos propostos concluiu-se que:

O estudo da caracterização mostrou que as cinzas volantes resíduos são constituídos por quartzo, mulita e hematita e o subproduto da FGD por sulfato de cálcio e traços de quartzo. A cinza pode ser considerada um material pozolânico.

A substituição de cimento Portland por $6 \%$ em massa de cinzas volantes aumenta a resistência a compressão axial da argamassa em 5\% assim, isso resulta em uma redução no consumo de cimento de 187,2 $\mathrm{kg} / \mathrm{m}^{3}$. Além disso, devido absorção de água foi menor nesta formulação.

A cinza volante melhora a durabilidade das argamassas devido ao consumo de hidróxido de cálcio $\left(\mathrm{Ca}(\mathrm{OH})_{2}\right.$ ou portlandita) e ao refinamento dos poros.

O tempo de cura tem interação com o traço de argamassa e com o teor de substituição.

Não foram evidenciadas melhorias nas argamassas com adições de SP.

A utilização das cinzas volantes utilizadas nesse estudo em substituição Portland, limitada ao teor de 6\% em massa, é uma opção viável para a indústria cimenteira, haja vista as vantagens econômicas e ambientais propiciadas. A redução no consumo de clínquer e a adequada destinação de resíduos industriais justificam a crescente utilização desse resíduo, pois insere o cimento Portland e a geração termoelétrica nos preceitos da sustentabilidade e fomenta a mitigação do passivo ambiental das empresas geradoras de resíduos.

\section{BIBLIOGRAFIA}

[1] TREVISOL, C.A., SILVA, P.R.P., PAULA, M.M.S., et al. "Avaliação de inibidores de corrosão para estruturas de concreto armado", Revista Matéria UFRJ, 22, n . 4, 2017.

[2] GASPARETTO, A., PANTOJA, J.C., RAMIRES, F.B. "Metodologia para inspeção e avaliação da segurança e durabilidade de estruturas de concreto armado", Brazilian Journal of Development, 7.1: 4942-4960. 2021.

[3] TINOCO, M.P., SILVA, F.A. "Viabilidade da aplicação de compósitos do tipo SHCC para melhoria da durabilidade de estruturas de concreto", Gestão e Gerenciamento, 13.13: 52-59. 2020.

[4] OLIVEIRA. J., SERRA, F.A., et al. "Análise da substituição do aço por bambu em estruturas de concreto armado", Brazilian Journal of Development, 6.9: 72453-72467. 2020.

[5] UENDE, S.B., SALOMÃO, P.E.A., LAUAR, G.T., RIBEIRO, P. T."Reutilização do concreto como contribuição para a sustentabilidade na construção civil", Revista Multidisciplinar do Nordeste Mineiro-Unipac, ISSN 2178: 6925. 2018.

[6] BARROS, E., FUCALE, S. "O Uso de Resíduos da Construção Civil como agregados na Produção de Concreto", Revista de Engenharia e Pesquisa Aplicada, v. 2, n. 1, 2016.

[7] CAPELIN, L.J., MORAES, K.K., ZAMPIERI, J.P, et al. "Avaliação dos Efeitos da Fibra de Coco e da Microcelulose Cristalina nas Propriedades de Argamassas Cimentícias", Revista Matéria UFRJ, v. 25, n. 1, 2020.

[8] PEREIRA, A.M., SILVA, C.A.R., QUEIROZ, D.C.A.M., et al. "Estudo das propriedades mecânicas do concreto com adição de cinza de casca de arroz",Revista Matéria UFRJ, v. 20, n. 1, 2015.

[9] SALES, A., LIMA, S.A. "Use of Brazilian sugarcane bagasse ash in concrete as sand replacement", Waste management, v. 30, n. 6, p. 1114-1122, 2010.

[10] MATOS, P.R., JUNCKES, R., PRUDÊNCIO, L.R. "Influência do uso de cinza volante na elevação adiabática de temperatura e resistência à compressão de concretos", Revista Matéria UFRJ, v. 24, n. 2, 2019.

[11] COSTA, F.L. "Influência do uso de aditivo acelerador de resistência baseado em nitrato de cálcio no desempenho de argamassas de cimento Portland com adição de cinza volante", Revista Matéria UFRJ, v. 23, n. 3, 2018 .

[12] KNIESS, C.T., COSTA, P.R., QUONIAM, L., et al. "Utilização do resíduo resultante da combustão de carvão mineral em usinas termelétricas na produção de novos materiais: uma análise a partir de artigos científicos e de patentes", Revista de Gestão social e ambiental, 13.1: 76-93. 2019.

[13] SALUM, P.L. "Efeito da elevação de temperatura sobre a resistência à compressão de concretos massa com diferentes teores de cinza volante", Dissertação de M.Sc, Universidade Federal de Santa Catarina, Cen- 
tro Tecnológico, Programa de Pós-Graduação em Engenharia Civil, Florianópolis. 2016.

[14] WITZKE, F.B., CORREIA, S.L., MEDEIROS, R.A. "Abrasão superficial de concretos contendo cinza volante em substituição parcial ao cimento Portland”, Revista Técnico-Científica, 21. 2019.

[15] FELTRIN, C.S., ISAIA, G.C. e LUBECK, A. "Efeitos sinérgicos entre adições minerais na resistência e microestrutura de concretos", IBRACON Structures and Materials Journal, 13.6. 2020.

[16] DOURADO, K.C.A. MOTA, J.M.F., BARBOSA, F.R., et al. "Influência da adição de pozolana em concretos moldados na região de Caruaru" In: $9^{\circ}$ Simpósio Internacional de estruturas, geotecnia y materiales de construción. Pernambuco, 2018.Anais [...]. Pernambuco, 2018.

[17] ALTHEMAN. D., FERREIRA, G., MONTINI, M., et al. "Avaliação de Cinza volante de carvão mineral em matrizes cimentícias”, Revista IBRACON de Estruturas e Materiais, 1320-1337, 2017.

[18] PACHECO, T.F., SHASAVANDI, A., JALALI, S. "Eco Efficient Concrete Using Industrial Wastes". A Review In: Materials Science Forum. p 581-586. Portugal. 2013.

[19] DUARTE, D., PELISSER, F., PETERSON, M. "Propriedades do concreto com adição de cinza volante e cinza pesada" In: $1^{\circ}$ Congresso Internacional de Tecnologias para o Meio Ambiente, Bento Gonçalves, 2008.

[20] SANTANA, R.A.A. "Avaliação do uso dos subprodutos da dessulfurização semi-seca dos gases da combustão da termelétrica do Pecém como insumo para a construção civil”, Dissertação M.Sc, Universidade Federal do Ceará, Fortaleza, 2018.

[21] ASSOCIAÇÃO BRASILEIRA DE NORMAS TÉCNICAS. NBR 5736: Cimento Portland Pozolânico. Rio de Janeiro: ABNT, 1991.

[22] ASSOCIAÇÃO BRASILEIRA DE NORMAS TÉCNICAS. NBR NM 18: Agregados - Determinação da composição granulométrica. Rio de Janeiro: ABNT, 2012.

[23] ASSOCIAÇÃO BRASILEIRA DE NORMAS TÉCNICAS. NBR NM 11-2: Cimento Portland - Análise química - Método optativo para determinação de óxidos principais por complexometria. Parte 2: Rio de Janeiro: ABNT, 2009.

[24] ASSOCIAÇÃO BRASILEIRA DE NORMAS TÉCNICAS. NBR NM 15: Cimento Portland - Análise química - Determinação de resíduo insolúvel. Rio de Janeiro: ABNT, 2012.

[25] ASSOCIAÇÃO BRASILEIRA DE NORMAS TÉCNICAS. NBR NM 13: Cimento Portland - Análise química - Determinação de óxido de cálcio livre pelo etileno glicol. Rio de Janeiro: ABNT, 2012.

[26] ASSOCIAÇÃO BRASILEIRA DE NORMAS TÉCNICAS. NBR NM 23: Cimento Portland - Análise química - Cimento Portland e outros materiais em pó - Determinação da massa específica. Rio de Janeiro: ABNT, 2001.

[27] ASSOCIAÇÃO BRASILEIRA DE NORMAS TÉCNICAS. NBR NM 76: Cimento Portland - Determinação da finura por meio da peneira $75 \mu \mathrm{m}\left(n^{\circ} 200\right)$ - Método de ensaio. Rio de Janeiro: ABNT, 1998.

[28] ASSOCIAÇÃO BRASILEIRA DE NORMAS TÉCNICAS. NBR 11579: Cimento Portland - Determinação da finura pelo método de permeabilidade ao ar (Método de Blaine). Rio de Janeiro: ABNT, 1991.

[29] ASSOCIAÇÃO BRASILEIRA DE NORMAS TÉCNICAS. NBR NM 43: Cimento Portland - Determinação da pasta de consistência normal. Rio de Janeiro: ABNT, 2003.

[30] ASSOCIAÇÃO BRASILEIRA DE NORMAS TÉCNICAS. NBR NM 65: Cimento Portland - Determinação do tempo de pega. Rio de Janeiro: ABNT, 2003.

[31] ASSOCIAÇÃO BRASILEIRA DE NORMAS TÉCNICAS. NBR 11582: Cimento Portland - Determinação da expansabilidade de Le Chatelier - Método de ensaio. Rio de Janeiro: ABNT, 2012.

[32] ASSOCIAÇÃO BRASILEIRA DE NORMAS TÉCNICAS. NBR 7215: Cimento Portland - Determinação da resistência à compressão. Rio de Janeiro: ABNT, 2019.

[33] ASSOCIAÇÃO BRASILEIRA DE NORMAS TÉCNICAS. NBR 7211: Agregados para Concreto Especificações. Rio de Janeiro: ABNT, 2005. 
[34] MALHERBE C., HUTCHINSON, I.B., McHUGH, H.L., et al. "Minerals and microstructure identification using Raman instruments: Evaluation of field and laboratory data in preparation for space mission", Journal of Raman Spectroscopy, v. 51, n. 9, p. 1613-1623, 2020.

[35] Database of Raman spectroscopy, X-ray diffraction and chemistry of minerals. Disponível em: https://rruff.info/. Acesso em 01 ago.2020.

[36] ASSOCIAÇÃO BRASILEIRA DE NORMAS TÉCNICAS. NBR 7181: Solo - Análise granulométrica. Rio de Janeiro: ABNT, 2018.

[37] ASSOCIAÇÃO BRASILEIRA DE NORMAS TÉCNICAS. NBR 9779: Argamassa e concreto endurecidos. Determinação da absorção de água por capilaridade, índice de vazios e massa específica: Método de ensaio. Rio de Janeiro: ABNT, 2012.

[38] HOPPE, F.J., GOBBI, A., QUARCIONI, V.A., et al. "Atividade pozolânica de adições minerais para cimento Portland (Parte I): Índice de atividade pozolânica (IAP) com cal, difração de raios-X (DRX), termogravimetria (TG/DTG) e Chapelle modificado", Revista Matéria UFRJ, v. 22, n³. 2017.

[39] YAN, K., GUO, Y., MA, Z.Z., et al. "Quantitative analysis of crystalline and amorphous phases in pulverized coal fly ash based on the Rietveld method", Journal of Non-Crystalline Solids, v. 483, p. 37-42, 2018.

[40] KRÓL, M., ROŻEK, P., MOZGAWA, W. "Synthesis of the Sodalite by Geopolymerization Process Using Coal Fly Ash", Polish Journal of Environmental Studies, v. 26, n. 6, 2017.

[41] AZEVEDO, A.G.S. STRECKER, K., BARROS, L.A., et al. "Effect of curing temperature, activator solution composition and particle size in brazilian fly-ash based geopolymer production", Materials Research, v. 22, 2019.

[42] PEREIRA, L.F.S. "Estudo da reciclagem de cinza volante para produção de agregado sintético utilizando reator de leito fixo", Dissertação M.Sc, Universidade Federal do Pará - Instituto de Tecnologia ITEC - Programa de Pós Graduação em Engenharia Química. Belém do Pará - PA. 2016.

[43] LI, J., et al. "Potential utilization of FGD gypsum and fly ash from a Chinese power plant for manufacturing fire-resistant panels", Construction and building materials. v. 95, p. 910-921, 2015.

[44] MIAO, M., FENG, XIN., WANG, GANGLING., et al. "Direct transformation of FGD gypsum to calcium sulfate hemihydrate whiskers: Preparation, simulations, and process analysis", Particuology, v. 19, p. 5359, 2015.

[45] GUPTA, V., PATHAK, D.K., DIDDIQUE, S., et al. "Study on the mineral phase characteristics of various Indian biomass and coal fly ash for its use in masonry construction products", Construction and Building Materials, v. 235, p. 117413, 2020.

[46] SINGH, N., SHEHNAZDEEP., BHARDWAJ, A. "Reviewing the role of coal bottom ash as an alternative of cement", Construction and Building Materials, v. 233, p. 117276, 2020.

[47] SUN, H., TAN, D., PENG, T., et al. "Preparation of calcium sulfate whisker by atmospheric acidification method from flue gas desulfurization gypsum", Procedia Environmental Sciences, 31, 621-626. 2016.

[48] CAILLAHUA, M.C., MOURA, F.J. "Technical feasibility for use of FGD gypsum as an additive setting time retarder for Portland cement", Journal of materials research and technology, 7.2: 190-197. 2018.

[49] MEHTA, P.K, MONTEIRO, P.J.M. "Concreto: microestrutura, propriedades e materiais", São Paulo: IBRACON, 2008.

[50] COSTA, A.B. “Potencial Pozolânico da Cinza Volante como Material de Substituição Parcial de Cimento", Trabalho de Conclusão de Curso apresentado ao Centro de Ciências Exatas e Tecnológicas da UNIVATES, 2008.

[51] LACERDA, L.V. "Síntese e caracterização de zeólita tipo sodalita obtida a partir de cinzas volantes de carvão mineral utilizado na usina termoelétrica de Candiota-RS", Dissertação de M.Sc, Escola de Engenharia, Universidade Federal do Rio Grande do Sul, Porto Alegre, 2015.

[52] DIAS, Y.R., RODRIGUES, R., MUNIZ, A.R.C. "Estudo da adsorção em leito fixo de cinzas volantes na remoção de compostos de enxofre do gás obtido na gaseificação do carvão mineral de Candiota-RS", In: $V$ 
Congresso Brasileiro de Carvão Mineral. Criciúma, 2017. Anais [...]. Criciúma, 2017.

[53] ZERBINO, R., GIACCIO, G., ISAIA, G.C. "Concrete incorporating rice-husk ash without processing”, Construction and Building Materials, v. 25, n. 1, pp. 371-378, January 2011.

[54] HOANG, K., JUSTNES, H., GEIKER, M., "Early age strength increase of fly ash blended cement by a ternary hardening accelerating admixture", Cement and Concrete Research, v.81, pp. 59-69, 2016.

[55] TASHIMA, M.M. Cinza de casca de arroz altamente reativa: método de produção, caracterização físicoquímica e comportamento em matrizes de cimento Portland, dissertação de M.Sc., FEIS/UNESP, Ilha Solteira, SP, Brasil, 2006.

[56] KHATIB, J.M., WRIGHT, L., MANGAT, P.S. "Mechanical and physical properties of concrete containing FGD waste”, Magazine of concrete Research, v. 68, n. 11, p. 550-560, 2016.

[57] MEDEIROS, J. R. A., MUNHOZ, G. S., MEDEIROS, M. H. F. “Correlations between water absorption, electrical resistivity and compressive strength of concrete with different contents of pozzolan", Revista ALCONPAT, 9.2: 152-166. 2019.

[58] ASSOCIAÇÃO BRASILEIRA DE NORMAS TÉCNICAS. NBR 16072: Argamassa impermeável. Rio de Janeiro: ABNT, 2012.

[59] OLSSON, N., BAROGHEL, B.V., NILSSON, L.O., et al. "Non-saturated ion diffusion in concrete - A new approach to evaluate conductivity measurements", Cement and Concrete Composites, 40:40-47. 2013.

[60] MEDEIROS, J.R.A., LIMA, M.G. "Electrical resistivity of unsaturated concrete using different types of cement", Construction and Building Materials, 107:11-16. 2016.

[61] BOTAS, S.M.S. “Avaliação do comportamento de argamassas em climas frios”, Dissertação de MSc., Universidade Nova de Lisboa, Faculdade de Ciências e Tecnologia-Lisboa, 2019.

\section{ORCID}

Daniele Ferreira Lopes

https://orcid.org/0000-0003-3321-9351

Sabrina Neves da Silva

https://orcid.org/0000-0002-2037-294X 